\title{
Ulrich Döring, Spurensuche. Kultur und kulturelle Identität in Driss Chra bis Berber-Trilogie
}

\section{Stefania Stefani}

\section{(2) OpenEdition}

1 Journals

\section{Edizione digitale}

URL: http://journals.openedition.org/studifrancesi/30867

DOI: 10.4000/studifrancesi.30867

ISSN: 2421-5856

\section{Editore}

Rosenberg \& Sellier

\section{Edizione cartacea}

Data di pubblicazione: 1 avril 2006

Paginazione: 197-198

ISSN: 0039-2944

Notizia bibliografica digitale

Stefania Stefani, «Ulrich Döring, Spurensuche. Kultur und kulturelle Identität in Driss Chra bis Berber-

Trilogie», Studi Francesi [Online], 148 (XLX | I) | 2006, online dal 30 novembre 2015, consultato il 18 avril 2021. URL: http://journals.openedition.org/studifrancesi/30867 ; DOI: https://doi.org/10.4000/

studifrancesi.30867

Questo documento è stato generato automaticamente il 18 avril 2021.

\section{(c) (1)}

Studi Francesi è distribuita con Licenza Creative Commons Attribuzione - Non commerciale - Non opere derivate 4.0 Internazionale. 


\title{
Ulrich Döring, Spurensuche. Kultur und kulturelle Identität in Driss Chra bis Berber-Trilogie
}

\author{
Stefania Stefani
}

\section{NOTIZIA}

ULRICH DÖRING, Spurensuche. Kultur und kulturelle Identität in Driss Chra bis Berber-Trilogie, Frankfurt/M.-Berlin-Bern-Bruxelles-New York-Oxford-Wien, Peter Lang, 2003, pp. 186.

1 Ulrich Döring (Leipzig, 1951), germanista e francesista, si addottora nel 1984 con una tesi sulla letteratura fantastica francese e ottiene il Prix Strasbourg. Fino al 1992 collabora alla cattedra di Romanistica dell'Università di Tübingen. Fra i suoi contributi alla ricerca è da menzionare uno studio sulla ricezione di Antoine Furetières (Peter Lang, 1995). Questo invece il titolo, reso in italiano, che la Döring sceglie per il presente studio critico, e che già si rivela efficace sunto delle problematiche ivi affrontate: Alla ricerca delle tracce. Cultura e identità culturale nella trilogia berbera di Driss Chraïbi. Considerato uno dei padri della moderna letteratura maghrebina, Chraïbi è noto soprattutto per avere creato il personaggio dell'ispettore Alì, eroe seriale dei suoi romanzi polizieschi. Prima di concentrarsi sull'analisi della "trilogia" ambientata in un Marocco ancestrale di contro all'isterilita modernità delle società occidentali, la studiosa ripercorre le varie fasi della produzione e del pensiero dell'autore. Il libro è anche arricchito da una dotta bibliografia sia sulla "storia delle sue opere", sia sulla "storia della critica e della fortuna", che è strumento prezioso per i lettori che vogliano avvicinarsi alla scrittura di Chraïbi, ancora troppo poco studiata.

Questi i romanzi della trilogia storico-epica, che la Döring ripercorre in una trattazione sistematica nei tre capitoli principali del volume: Une Enquête au pays (1981), La Mère du printemps (1982) e Naissance à l'aube (1986). Tali opere rappresentano il compimento di un progetto davvero singolare, forse unico nella letteratura del Maghreb, che consiste 
nell'esplorazione della cultura delle popolazioni berbere, in particolare dei tempi delle loro origini. La monografia della Döring è incentrata sull'analisi di quelle che la studiosa individua come le due linee guida della tematizzazione di questa cultura compiuta da Chraïbi: 1- una duplice prospettiva temporale orientata prima sul passato in quanto serbatoio di una memoria collettiva cui attingere per poi edificare un rinnovato presente dell'etnia berbera; 2-il proposito di fare chiarezza sul crogiolo di relazioni e conflitti che lega tanto agli Arabi quanto ai Francesi il popolo berbero, che in tutte le fasi della sua storia non ha mai cessato di lottare per le proprie rivendicazioni identitarie, per vedere riconosciuto il posto che gli spetta nella vita dei rispettivi paesi. È infatti l'"essenza" originaria di questa antica civiltà orientale che Chraïbi si propone di indagare all'insegna di un'auspicata interpenetrazione pacifica fra culture diverse. Leitmotiv della trilogia (ma anche di tutta la sua scrittura) è la questione-ginepraio dell'interculturalità come rapporto fra civilisation e acculturation, ossia del complesso fenomeno di una possibile, ma pur sempre problematica, assimilazione berbera (e maghrebina tout court) all'“occidentalità" e alla modernità.

3 Con le opere degli anni Cinquanta (Le passé simple e Les boucs) Chraïbi era stato anticipatore del "nuovo", descrivendo per la prima volta la quotidianità dei lavoratori immigrati, nella cornice di una Francia ancora connotata come terra di "bonheur", e di una società marocchina che egli giudicava in modo negativo, come ipocrita, eccessivamente ancorata al passato e chiusa entro un rozzo egocentrismo. È a partire dagli anni Sessanta che l'autore mette in discussione il modello occidentale, guardandolo d'ora in poi con gli occhi del maturo disincanto, poiché consapevole dell'inevitabile "prezzo" della "civilisation". Ed è proprio con la redazione della trilogia che Chraïbi, ormai convinto di non riuscire ad essere «écrivain maghrébin d'expression française», si pone alla ricerca delle tracce della cultura berbera e nel contempo della propria identità di écrivain "soltanto" français. Il sogno che serpeggia come filo rosso dei tre romanzi è quello di assistere, un giorno, al compiersi di una "acculturation" che non sia più assimilazione distruttiva ma che, al contrario, sia integrazione e interscambio fra culture, in virtù dei valori puri ed originari del Maghreb.

4 Chraibi illustra in un modo del tutto nuovo e mai sperimentato in alcun testo letterario la tradizionale dicotomia Oriente/Occidente: in questo caso, come osserva anche la Döring, lo scontro fra mondi diversi è affrontato dall'interno, nel senso cioè che il tema conduttore è il conflitto, tutto orientale e intestino, fra i Berberi (preposti a simbolo dell'Oriente, invaso e oppresso) e gli Arabi (preposti a simbolo dell'Occidente, invasore e oppressore). La tematizzazione di tale conflitto diventa nel contempo occasione per l'autore di costruire una fenomenologia sociologico-letteraria dei tratti peculiari delle due etnie: poiché per Chraïbi l'assimilazione non deve mai naufragare nello straniamento, né tanto meno nella perdita delle proprie origini, bensì in un loro potenziamento. Ampio spazio viene dedicato dalla Döring a Une enquête au pays, il testo che a suo parere problematizza in maniera più pregnante questa insolita forma di contrapposizione fra civiltà, sullo sfondo di un Marocco contemporaneo che guarda fiducioso verso un ritorno ai tempi della cultura prearaba, concepita e difesa come la "vera" cultura maghrebina, originale e originaria.

5 La particolarità degli altri due romanzi risiede invece nel fatto di spostare l'indagine indietro nella Storia berbera, all'epoca della conquista araba del Nordafrica e della Spagna. La prima parte di Mère du printemps è un'apologia del modo di vita berbero, caratterizzato da uno stretto legame con la natura e da una radicata coscienza di sé, di 
chi lotta non per conquistare beni o territori, ma per proteggere la propria cultura. In questo romanzo la cultura berbera diventa simbolo portatore del nuovo concetto elaborato da Chraïbi di una civilisation "unificante", secondo il quale, cioè, l'opposizione fra le sfere islamico-araba e cristiano-europea non ha più ragione di esistere. Di Naissance à l'aube la studiosa apprezza il valore simbolico del titolo: la pregnanza del romanzo è data, infatti, dalla felice riconciliazione, non priva tuttavia di slanci utopistici, di ogni sorta di conflitto espressa dalla tenera allegoria della doppia "nascita all'alba" di un bimbo berbero e del figlio dell'emiro.

Uno degli spunti più originali di questo volume critico risiede nell'accurata disamina della ricezione della trilogia, che a più riprese sottolinea l'audacia di Chraïbi nell'addentrarsi in questa tematica, avvertita come un autentico tabù, poiché negli anni Ottanta la cultura ufficiale e l'opinione pubblica consideravano il conflitto tra Berberi e Arabi da lungo tempo ormai risolto. In tal senso, questa monografia ridesta l'attenzione verso queste problematiche, ri-semantizzandole attraverso gli occhi di Chraïbi. In conclusione, questo volume di Ulrich Döring si rivela un pregevole contributo alla critica non soltanto perché vivifica gli studi su Driss Chraïbi e sui Berberi, ma soprattutto perché permette di cogliere il ruolo primario della scrittura di questo autore. La trilogia epica è in questo senso guardiana della memoria del "passato" berbero e di una sua applicabilità in un "presente" che consenta di far emergere la pars construens del conflitto fra le due etnie, mettendo in risalto ciò che le accomuna, e facendo sì che ogni differenza venga concepita come un'apertura (soprattutto mentale) all'étranger, e sia dunque reciproco arricchimento, in primis umano. La Döring perviene così a un interessante ampliamento del concetto di civilisation e ad una sua risemantizzazione, che grazie alla riflessione sulla trilogia di Chraïbi non si riferisce più semplicemente a un'assimilazione forzata che annienta le origini, una forma, cioè, di cultura dell'oppressione: esso diventa ora sinonimo di una trans-culturalità in cui l'“io" accoglie il "tu". È il contatto con la memoria dei tempi prearabi che permette all'autore di ricostruire nei tre romanzi una rinnovata identità berbera, che è al tempo stesso scoperta di una nuova possibile esperienza di libertà: e, non a caso, l'antica espressione con la quale i Berberi definivano se stessi è "Amazigh", "uomo libero". Quello che Chraïbi affida alla "trilogia berbera" è un messaggio luminoso di speranza per un ritorno a questa arcaica cultura depositaria di un'originarietà "pura", che la scrittura riesce a sottrarre all'oblio e all'indifferenza. E si percepisce intensa l'emozione della Döring, che contribuisce insieme all'autore a recuperare la memoria di questo popolo culla delle civiltà maghrebine: è l'emozione che nasce ogni volta in cui si assiste alla riattivazione di un mito. 